\title{
Role of HLA-A, HLA-B, HLA-DRB1 and HLADQB1 Alleles in HIV-1 Patients with Pulmonary Tuberculosis Co-Infection from Western India
}

\section{U. Shankarkumar*, A. Shankarkumar}

National Institute of Immunohaematology, ICMR, KEM Hospital, Mumbai, India.

E-mail: "shankar2kumar@rediffmail.com

Received August 20 ${ }^{\text {th }}$ 2011; revised September 27 ${ }^{\text {th }}, 2011$; accepted October $14^{\text {th }}, 2011$.

\begin{abstract}
We attempted to study the role of HLA HLA-A, B, DRB1 and DQB1 in HIV-1 patient's co infected with pulmonary tuberculosis (PTB). A total of $102 \mathrm{HIV}-1+$ patients co-infected with pulmonary tuberculosis and 200 healthy controls were included in HLA analysis. HLA-A*, HLA-B* HLA-DRB1* and DQB1* typing was done molecularly by PCRSSOP (Polymerase Chain reaction-Sequence Specific Oligonucleotide Probing) method using kit (Dynal Kit-Invitrogen). The frequencies of the HLA-A, B HLA-DRB, 1 and DQB1 alleles were determined using standard software. The HLA alleles identified among $H I V+v e / P T B+v e$ co-infected patients as compared with healthy controls showed a significantly increased frequency of HLA-B*08:01:01 in $H I V+v e / P T B+v e$ co-infected patients when compared with healthy controls ( $p=0.011$, OR 3.335, 95\% CI 1.35 - 8.18), Likewise HLA-DQB1*03:01:03 was significantly increased in HIV+ ve/PTB+ve co-infected patients as against healthy controls ( $p<0.0001$, OR 107.5, 95\% CI 6.195 - 1865.3). Similarly $H L A-D Q B * 06: 01: 02$ allele frequency was observed in HIV + ve/PTB + ve co-infected patients as against healthy controls ( $p=0.003$, OR 4.808, 95\% CI 1.72 - 13.39), HLA-DQB1*03:01:01 ( $p=0.045$, OR 0.219, 95\% CI 0.051 - 0.940), HLA-DQB1*06:01:01:01 ( $p=0.012$, OR 0.334, 95\% CI 0.145 - 0.770), alleles in HIV+ve/PTB+ve co-infected patients when compared with healthy controls. We can be concluded that different HLA alleles may render susceptibility or protection to in different ethnic population.
\end{abstract}

Keywords: HIV1, PTB, Co-Infection, HLA, Western India

\section{Introduction}

Acquired immunodeficiency syndrome (AIDS) caused by HIV infection is endemic all over the globe and it is on the rise especially in resource limited countries. Over 33 million people are living with HIV, 2.5 million are newly infected and 2.1 million people have died of AIDS [1]. Individuals with impaired cell mediated immunity due to AIDS have a greatly increased risk of co-infection with Mycobacterium tuberculosis [1-2]. The co-infection of HIV-1 and Mycobacterium tuberculosis causes two infectious diseases endangering human health significantly. The pathogenesis of HIV-1 and PTB co-infection is unavailable. The factors influencing the greater inter individual variability to susceptibility to PTB co-infection and progression of AIDS is yet to be identified. This may be due to considerable varied immune re- sponses of HIV-1 and MTB exposed individuals may result from the different genetic background. MHC class-I restricted CD8+ T cells are important for the generation of protective immune response in Mycobacterium tuberculosis infection. CD8+ CTL (Cytotoxic T lymphocytes)—derived IFN- $\gamma$ may be especially important both for cells lacking MHC class-II molecules.

\section{Materials and Methods}

Both HLA Class I and class II genes have been shown to be associated with susceptibility or resistance to HIV infection [3]. Among the HLA Class-II alleles, HLADQB1 and HLA-DBP1 alleles have shown to be associated with HIV infection [4,5]. However, we have much less information about the HLA linked genetic control of susceptibility to HIV-1 and MTB co-infection. We have 
attempted to study the role of HLA-A, B,DRB1 and DQB1 in HIV-1 patients with pulmonary tuberculosis (PTB). A total of 102 HIV-1+ patients co-infected with pulmonary tuberculosis and 200 healthy controls were included in HLA analysis. HLA typing was done molecularly by PCR-SSOP (Polymerase Chain reaction-Sequence Specific Oligonucleotide Probing) method using kit (Dynal Kit-Invitrogen). The frequencies of the HLAA, B, HLA-DRB1 and DQB1 alleles were determined by using standard software.

\section{Results}

The HLA class HLA and I class II alleles identified among HIV+ve/PTB+ve co-infected patients as compared with healthy controls are given in Table 1. Significantly increased frequency of HLA-B*08:01:01 was observed in HIV+ve/PTB+ve co-infected patients when compared with healthy controls $(\mathrm{p}=0.011$, OR 3.335, 95\% CI 1.35 - 8.18) Likewise HLA-DQB1* 03:01:03 was significantly increased in $\mathrm{HIV+ve/PTB+ve} \mathrm{co-in-}$ fected patients as against healthy controls $(\mathrm{p}<0.0001$, OR 107.5, 95\% CI 6.195 - 1865.3). Similarly HLADQB*06:01:02 allele frequency was observed in HIV+ ve/PTB+ve co-infected patients as against healthy controls ( $\mathrm{p}=0.003$, OR 4.808, 95\% CI 1.72 - 13.39), A significantly increased frequency of HLA-A*02:11 ( $\mathrm{p}=$ 0.015, OR 1.762, 95\% CI 1.13 - 2.73), HLA-B* 57:01:01(p = 0.017, OR 1.973, 95\% CI $1.15-3.37)$, HLA-B*56:01:01 ( $=0.029$, OR 2.606, 95\% CI $1.16-$ 5.85), HLA-DRB $1 * 040301(\mathrm{p}=0.006$, OR $7.727,95 \%$ CI 1.79 - 33.3), HLA-DRB1*09:01:02 ( $\mathrm{p}=0.012$, OR 9.143, 95\% CI 1.63 - 51.174), HLA-DRB1*14:01:03 (p $=0.024$, OR 13.526, 95\% CI $1.381-132.49$ ), HLA-
DQB1*05:02:01 ( $<$ 0.0001, OR 28.556, 95\% CI 8.36 242.16), and a significantly decreased frequency was observed in HLA-B*51:01:01 ( $=0.009$, OR 0.434, 95\% CI $0.236-0.799)$, HLA-DQB1*03:01:01 ( $\mathrm{p}=0.045$, OR 0.219, 95\% CI 0.051 - 0.940), HLA-DQB1*06:01:01:01 $(\mathrm{p}=0.012$, OR $0.334,95 \%$ CI $0.145-0.770)$, alleles in HIV+ve/PTB+ve co-infected patients when compared with healthy controls.

\section{Discussion}

We have studied, HLA-A, B, DRB and DQB loci to find out the role of these HLA alleles in HIV+ve/PTB+ve co-infection. Significantly increased frequency of HLA$\mathrm{B} * 08: 01: 01$ and HLA-DQB*03:01:03 in HIV+ve/PTB+ ve co-infected patients against controls may suggest that, these alleles play an associative role in HIV infection and PTB development. Our study reveals that, HLA-B*08:01:01and HLA-DQB*03:01:03 are associative to enhance HIV infection. The decreased frequency of HLA-DQB1*03:01:01 has been reported in PTB patients from China [6]. In our study, HLA-DQB1* 03:01:01 is decreased in HIV+ve/PTB+ve co-infected patients compared to controls suggesting that it may play a protective role in $\mathrm{HIV}+\mathrm{ve} / \mathrm{PTB}+\mathrm{ve}$ co-infection. Whereas HLA-DQB*03:01:03 is associative in HIV. Among South Indians an increased frequency of HLADQB1*06:01:01:01 has been reported in HIV-ve $\mathrm{PTB}+\mathrm{ve}$ and $\mathrm{HIV+ve} \mathrm{PTB}+\mathrm{ve}$ patients, suggesting that HLA-DQB1 ${ }^{* *} 06: 01: 01: 01$ is associated with susceptibility to PTB as well as development of PTB in HIV patients [7]. Further earlier association of HLA-DQB1* 06:01:01:01 with susceptibility to PTB has also been reported in south India [8]. In contrast to the above two

Table 1. Significant HLA alleles identified among the TB co-infected HIV patients from Western India.

\begin{tabular}{|c|c|}
\hline HIV + PTB $+(n=102)$ vs controls $(n=200)$ & HIV + PTB $+(n=102)$ vs controls $(n=200)$ \\
\hline Increased frequency & Decreased frequency \\
\hline HLA-B*08:01:01 & HLA-B*51:01:01 \\
\hline HLA-B*55:01:01 & HLA-DQB1*03:01:01 \\
\hline HLA-B*57:01:01 & HLA-DQB1*06:01:01 \\
\hline \multicolumn{2}{|l|}{ HLA-A*02:11 } \\
\hline \multicolumn{2}{|l|}{ HLA-DRB1*04:03:01 } \\
\hline \multicolumn{2}{|l|}{ HLA-DRB1*09:01:02 } \\
\hline \multicolumn{2}{|l|}{ HLA-DRB1*14:01:03 } \\
\hline \multicolumn{2}{|l|}{ HLA-DQB1*03:01:03 } \\
\hline \multicolumn{2}{|l|}{ HLA-DQB1*06:01:02 } \\
\hline HLA-DQB1*05:02:01 & \\
\hline
\end{tabular}


studies on South Indian population, it is reported that HLA-DQB1*06:01:01:01 plays a protective role against HIV disease progression in Europeans [9]. In our study a significantly increased frequency of HLA-DQB*06:01: 02 in $\mathrm{HIV+ve/PTB+ve} \mathrm{co-infected} \mathrm{patients} \mathrm{when} \mathrm{com-}$ pared to controls and HIV+ve PTB-ve patients may suggest its strong association with both HIV infection and PTB co-infection. On the contrary HLA-DRB1* 06:01:01:01 frequency was significantly decreased in $\mathrm{HIV}+\mathrm{ve} / \mathrm{PTB}+$ ve co-infected patients compared to controls, thereby may protect from HIV infection and PTB development. HLA-DQB1*05:02:01 allele is reportedly related to high risk of developing TB in population from Asia and Latin America [10]. In the present study, frequency of HLADQB1*05:02:01 is increased in HIV+ve/PTB+ve coinfected patients compared to healthy subjects to show that HLA-DQB1* 05:02:01 may be associated with $\mathrm{HIV+ve/PTB+ve} \mathrm{co-infection.} \mathrm{There} \mathrm{was} \mathrm{no} \mathrm{consider-}$ able change in frequency of HLA-A*26:01:01 in HIV+ ve/PTB+ve co-infected patients when compared with controls and decreased frequency was observed when compared with HIV+ve PTB-ve patients. This suggests that, HLA-A*26:01:01 may not be associated with HIV infection and may play a protective role in PTB development. HLA-A*31 and HLA-B*41 antigens and the HLA-DRB1*10 and HLA-DQB1*05 were over represented in Brazilian patients with AIDS and tuberculosis, suggesting association to tuberculosis with AIDS [11]. As reported earlier HLA-DRB1*13 is associated with susceptibility to HIV-1 infection whereas HLA- DQB1*02:03 and DRB1*01 are resistant to HIV-1 infection which may vary in different ethnic groups [12]. HLA-DRB1*15 is susceptible in PTB development and DRB1*11 may be protective allele in Chinese population [6]. In the present study, HLA-DRB alleles HLA-DRB1*04:03:01, DRB1* 09:01:02, DRB1*14:01:03 and HLA-DQB1*05:02:01 allele are significantly increased in HIV+ve/PTB+ve co-infected patients compared to healthy controls. Thus, these alleles may be associated to susceptibility of HIV+ ve/PTB+ve co-infection among Indians.

\section{Conclusions}

It can be concluded that different HLA alleles may render susceptibility or protection to an infection in different ethnic population. HLA alleles may influence immunopathogenesis from either HIV and/or PTB infection. Further study on the HIV progression and resistant TB would enlighten the mechanism of action of the HLA in HIV and PTB infection.

\section{REFERENCES}

[1] Ministry of Health and Family Welfare, "National AIDS
Control Organization (NACO) Report 2008,” Government of India News Letter, 2008.

[2] V. Vijayalakshmi, S. R. Shilpa, B. Anuradha, et al., "Role of HLA-B51 and HLA-B52 in Susceptibility to Pulmonary Tuberculosis," Infection, Genetics and Evolution, Vol. 6, No. 6, 2006, pp. 436-439. doi:10.1016/j.meegid.2006.02.002

[3] M. Carrington and S. J. O’Brien, “The Influence of HLA Genotype on AIDS," Annual Review of Medicine, Vol. 54, 2003, pp. 535-551.

doi:10.1146/annurev.med.54.101601.152346

[4] A. P. Achord, R. E. Lewis, M. N. Brackin, et al., "HIV-1 Disease Association with HLA-DQ Antigen in Africans Americans and Caucasians," Pathobiology, Vol. 64, No. 4, 1996, pp. 204-208. doi:10.1159/000164049

[5] N. Odum, J. Georgsen, L. Fugger, et al., "HLA-DP Antigens in HIV Infected Individuals,” Disease Markers, Vol. 8, No. 3, 1990, pp. 113-116.

[6] J. Wang, C. Song and S. Wang, "Association of HLADRB1 Genes with Pulmonary Tuberculosis,” Chinese Journal of Tuberculosis and Respiratory Disease, Vol. 24, No. 5, 2001, pp. 302-305.

[7] P. Selvaraj, S. Ragavan, S. Swaminathan, et al., "HLADQB-1 and HLA-DPB-1 Allele Profile in HIV Patients Infected with or without Pulmonary Tuberculosis of South India," Infection, Genetics and Evolution, Vol. 8, No. 5, 2008, pp. 664-671.

doi:10.1016/j.meegid.2008.06.005

[8] M. Ravikumar, V. Dheenadhayalan, K. Rajaram, et al., "Associations of HLA-DRB1, DQB1 and DPB1 Alleles with Pulmonary Tuberculosis in South India,” Tubercle and Lung Disease, Vol. 79, No. 5, 1999, pp. 309-317. doi:10.1054/tuld.1999.0213

[9] A. Vyakarnam, D. Sidebottom, S. Murud, et al., "Possession of Human Leucocytes Antigen DQ6 Alleles and the Rate of CD4 T-Cell Decline in Human Immunodeficiency Virus-1 Infection,” Immunology, Vol. 112, No. 1, 2004, pp. 136-142. doi:10.1111/j.1365-2567.2004.01848.x

[10] A. Dubaniewicz, G. Moszhowska and Z. Szczerkowska, "Frequency of DRB1-DQB1 Two Locus Haplotypes in Tuberculosis: Preliminary Report,” Tuberculosis, Vol. 85, No. 4, 2005, pp. 259-267. doi:10.1016/j.tube.2004.12.003

[11] J. F. Fernando de castro, V. R. Maria de Lourdes, S. D. Neifi Hassam, et al., "HLA Profile in Patients with AIDS and Tuberculosis," The Brazilian Journal of Infectious Diseases, Vol. 12, No. 4, 2008, pp. 278-280.

[12] M. Patricia, M. Karina, S. Adrian, et al., "Association of HLA-DQ and HLA-DR Alleles with Susceptibility or Resistance to HIV-1 Infection among Population of Chaco Province, Argentina,” Medicina, Vol. 62, No. 3, 2002, pp. 245-248. 